\title{
Physics of Astroparticles
}

\author{
S. J. Sciutto \\ Departamento de Física and IFLP (CONICET) \\ Universidad Nacional de La Plata, Argentina
}

Received on 25 August, 2006

\begin{abstract}
The measurement of the properties of the highest energy astroparticles that hit the Earth's atmosphere is a challenging problem that the Auger experiment tries to solve. In this talk we present a general description of several aspects of the interactions between those high energy particles and the Earth's atmosphere, focusing in primary reconstruction. Special attention is dedicated to work done in our group regarding analysis performed with the help of air shower simulations.
\end{abstract}

Keywords: Astroparticles; Air shower Simulations; Auger experiment

\section{INTRODUCTION}

The existence of astroparticles or Cosmic Rays, that hit the Earth's atmosphere was first revealed by Victor Hess in 1912 [1]. From the 1930s to the 1950 s, before man-made particle accelerators reached very high energies, cosmic rays served as a source of particles for high energy physics investigations, and led to the discovery of subatomic particles that included positrons, muons and pions. It was also in that period when Pierre Auger demonstrated that some of the cosmic rays could generate particle showers containing thousands of secondary particles [2].

In 1962 a cosmic ray detector located in Volcano Ranch (NM, USA) recorded an extraordinary event [3]: a shower whose primary energy, $10^{20} \mathrm{eV}$, was significantly larger than the highest energies ever registered for such kind of event.

After that discovery, several air shower observatories have been used to study the properties of the highest energy cosmic rays. Even today, the measurement of the main characteristics of those particles continues to be a challenging issue, with three main questions pending closed answer:

- What are these particles?

- How are they accelerated?

- Where do they come from?

The aim of this paper is to review some of the current efforts to give answer to these questions. In the next session we will discuss the experimental approach, while in the remaining ones we will address several issues related to the research work that is being done at our group.

\section{THE AUGER OBSERVATORY}

The Pierre Auger Observatory [4] has been conceived to measure the flux, arrival direction, distribution and mass composition of cosmic rays from $10^{18} \mathrm{eV}$ to the very highest energies with high statistical significance over the whole sky. To achieve this coverage, the Observatory will have instruments located at two sites, one on each of the Northern and Southern Hemispheres. The astrophysical interest in this energy range is well known, stemming largely from the expectation of spectral features in the decade above $10^{19} \mathrm{eV}$.

Above $10^{20} \mathrm{eV}$, the rate of events is about 1 per square kilometer per century, so that vast areas must be monitored to collect a large statistical sample. The Pierre Auger Observatory has been planned as a pair of detector arrays, each of 3000 $\mathrm{km}^{2}$. The design for the Southern Observatory calls for 1600 water-Cherenkov detectors, arranged on a triangular grid, with the sides of the triangles being $1500 \mathrm{~m}$, overlooked from four sites by optical stations designed to detect air-fluorescence light. The water tanks respond to the particles reaching ground level (mainly muons, electrons, positrons, and photons), and the fluorescence cameras measure the emission from atmospheric nitrogen, which is excited by the charged particles of the shower as they traverse the atmosphere.

Presently, the Southern Hemisphere Observatory is almost complete, and data acquisition y currently operative. The fist preliminary results coming from the Auger Observatory have already been presented and published [5]. The most important ones regard the first estimation of the cosmic ray energy spectrum [6], the analysis of arrival directions in the search of anisotropies [7], and setting upper limits to the fraction of cosmic rays that could be photons [8].

\section{AIR SHOWER SIMULATIONS}

When a cosmic ray enters the Earth's atmosphere, it generates a shower of particles whose characteristics can be measured experimentally. The processes that take place during the shower development are very complex and make it almost impossible to obtain analytical relations between the properties of the primary particle and the measurable observables. Computer simulations are thus needed to properly analyze the experimental data.

In our group at La Plata University, we have been working in the development of a complete system for air shower simulation and analysis for nearly 10 years. As a result, we have designed, developed and tested the AIRES system for air shower simulations, a software package that is currently used by many scientists worldwide.

The AIRES simulation engine [9] provides full space-time particle propagation in a realistic environment, taking into ac- 
count the characteristics of the atmospheric density profile (using the standard US atmosphere), the Earth's curvature, and the geomagnetic field.

The following particles are taken into account in the AIRES simulations: photons, electrons, positrons, muons, pions, kaons, eta mesons, lambda baryons, nucleons, antinucleons, and nuclei up to $Z=36$. Nucleus-nucleus, hadronnucleus and photon-nucleus inelastic collisions with significant cross-sections are taken into account in the simulation. The hadronic processes are simulated using different models, accordingly to the energy: high energy collisions are processed invoking an external package (SIBYLL 2.1 [12], QGSJET01 [13] or QGSJETII [14]), while low energy ones are processed using an extension of Hillas splitting algorithm (EHSA) [9-11]. The threshold energies separating the low and high energy regimes used in our simulations are $200 \mathrm{GeV}$ and $80 \mathrm{GeV}$ for the SIBYLL and QGSJET cases, respectively. The EHSA low energy hadronic model used in AIRES is a very fast procedure, effectively emulating the major characteristics of low energy hadronic collisions. The model is adjusted to retrieve similar results as the high energy hadronic model for energies near the transition thresholds previously mentioned, and the low energy cross sections are calculated from parameterizations of experimental data.

AIRES has been successfully used to study several characteristics of high energy showers, including comparisons between hadronic models [11], influence of the LPM effect [15], muon bremsstrahlung [16], and geomagnetic deflections [17] on the shower development. AIRES has also been used to obtain an energy calibration of the AGASA experiment [18], and to study the expected efficiency of the Auger Observatory for detecting quasi-horizontal showers generated by $\tau$-neutrinos [19].

\section{PRIMARY AND SHOWER CHARACTERISTICS}

There are several shower observables that are relevant to determine the most important characteristics of the primary particle: direction of arrival, energy, and mass (composition).

In this section we review the essentials of the procedures used in a hybrid detector, like the Auger Observatory, to estimate the mentioned primary properties from the experimental data that can be taken at each event.

\section{A. Direction of arrival}

Both the Surface Detector (SD) and the Fluorescence Detector (FD) can easily determine the direction of arrival of a shower.

In the case of SD, the accurate GPS timing of each local station allows for adequate reconstruction of the shower front surface. The normal to this surface is precisely the shower axis that gives the direction of motion of the primary particle.

The light tracks detected by the FD can be used for an accurate determination of the so called shower-detector plane, that is, the plane that contains the shower axis and the FD that recorded the corresponding track. If two or more FD detectors recorded simultaneously the same event (stereo detection), then the shower axis can easily be determined as the intersection of all shower-detector planes. On the other hand, when a shower triggers a single FD detector (monocular detection), the shower axis cannot be always determined unambiguously. In such cases additional information, for example time of arrival of the shower front to a given SD station, is needed to remove the ambiguity.

For most of the events, the primary arrival direction is the observable that can be measured with best accuracy. Typical error figures are below 1 degree.

\section{B. Primary energy}

The estimation of the primary energy from the measurements performed by a SD is usually done by means of the so called lateral distribution, $S(r)$, that corresponds to the SD signal size measured at a distance $r$ from the shower axis. The SD primary energy estimation is based on the determination of $S\left(r_{0}\right)$, for a given distance $r_{0}$ that depends on the characteristics of the surface array, such as distance between stations, etc [20].

At any event, the SD measures signals at a discrete set of distances. The lateral distribution is then obtained after fitting a given function, with some free parameters, to the measured data [21]. Then $S\left(r_{0}\right)$ can be evaluated straightforwardly, and the primary energy can be evaluated using the following equation:

$$
\log E_{\text {prim }}=A \log S\left(r_{0}\right)+B
$$

The constants $A$ and $B$ depend on the characteristics of the detectors, and on some properties of the shower that need to be modeled via computer simulations. In particular, these parameters depend on the inclination of the shower [21], to the point that equation (1) cannot be used for accurate estimations of the primary energy when the zenith angle is larger than 70 degrees [22].

In figure 1 we show a typical Auger SD event. The dots correspond to the signals at the detectors triggered during the event, and the full line represents the best fit of the lateral distribution. In the Auger SD $r_{0}=1000 \mathrm{~m}$, and both $r_{0}$ and $S\left(r_{0}\right)$ are shown in the plot.

The FD allows to measure the primary energy from an estimation of the energy that the charged secondaries deposit in the atmosphere during the development of the shower [23]. An interesting point is that the FD energy estimation is an absolute measurement of the energy deposited by the electromagnetic shower that constitutes a lower bound of the true primary energy. The total primary energy is the sum of the deposited energy plus the so called "hidden energy" carried away by particles not related with the fluorescence emission mechanism, like neutrinos, for example. This hidden energy must be estimated from numerical simulations.

The FD energy measurement is essential for the cross calibration of a hybrid (SD + FD) detector [24]. Nevertheless, there exist a nontrivial set of aspects that could contribute to 


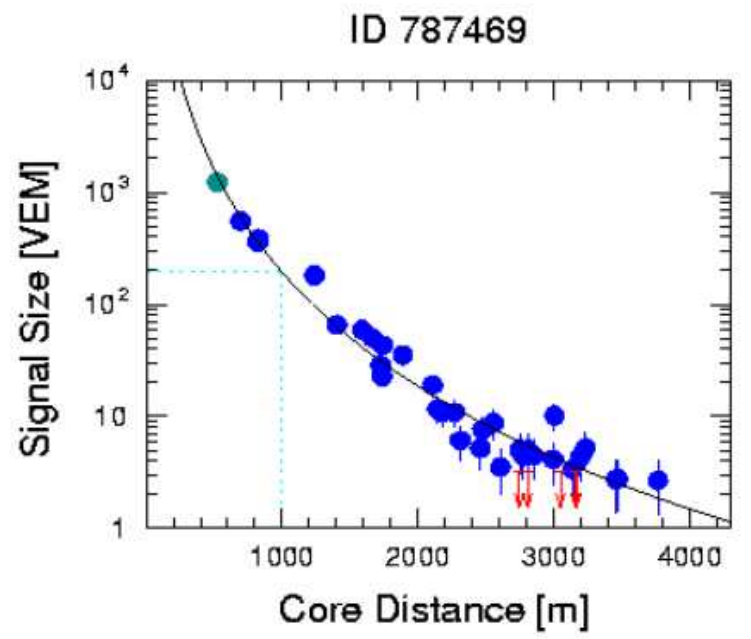

FIG. 1: Signal lateral distribution of a typical Auger Observatory event. The dots correspond to the actual signals measured by the triggered local stations, while the solid line represents a fit to an adequately chosen function. The dashed lines indicate the signal estimation for a distance of $1000 \mathrm{~m}$ from the shower axis.

enlarge the systematic errors that affect the estimated primary energy, in particular: the fluorescence yield that relates energy deposit with amount of fluorescence light emitted; Cherenkov light whose contribution must be subtracted from the detected signal [25]; atmospheric absorption and scattering, strongly dependent on the atmospheric conditions, that must be monitored continuously during the FD operation periods [26].

\section{Primary composition}

The estimation of the nature of a primary particle from the measurement of the properties of the air shower it generates after entering the Earth's atmosphere is one of the most challenging problems in experimental cosmic ray physics [4]. In the case of a hybrid observatory like the Auger Observatory, there are several methods that can be used for this purpose. In all cases, there is a remarkable dependence of the estimations on theoretical models used in the simulations that are needed to be able to interpret the experimental data.

The most common composition estimator is the depth of the shower maximum, $X_{\max }$. It is well known that there exists a direct correlation between the composition of the primary and the mentioned observable [27]. In the case of an hadronic primary, like a proton or a nucleus, and at a given primary energy, $X_{\max }$ diminishes when the mass of the primary is enlarged, allowing for a more or less clear separation of the expected extremes: proton or iron nuclei. A typical plot of $X_{\max }$ versus primary energy is displayed in figure 2 . The results coming from AIRES simulations for the cases of proton and iron primary are also included. Comparison from experimental and simulated data suggests that the cosmic rays

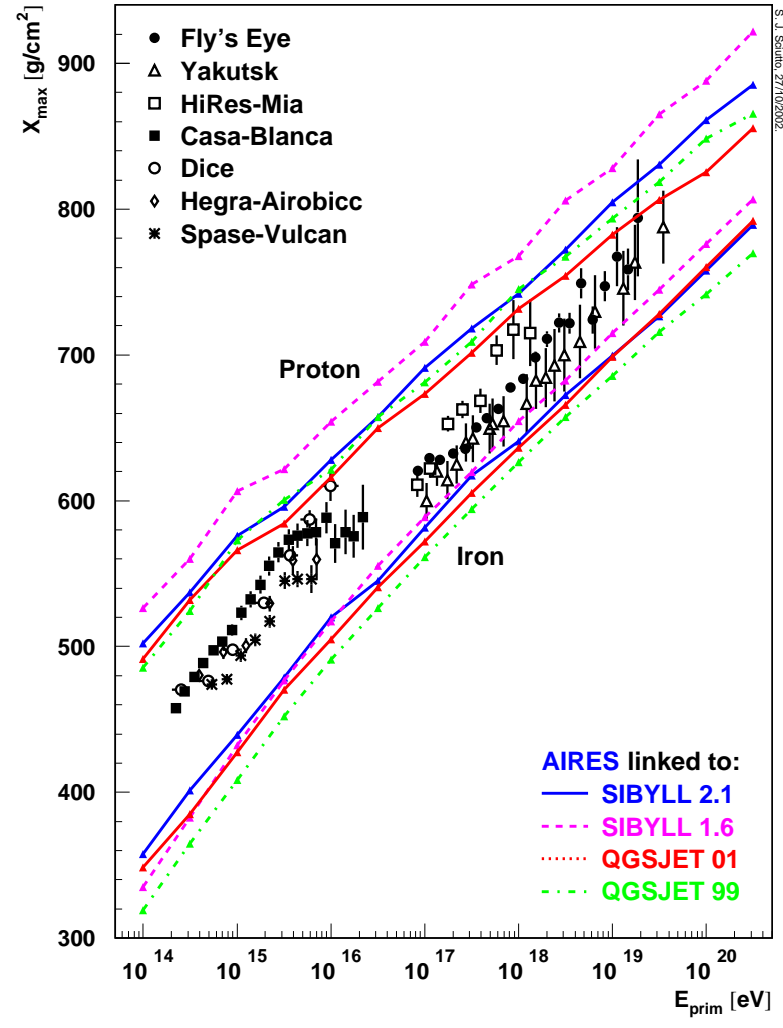

FIG. 2: Shower maximum versus primary energy

in that energy range could be a mixture of nuclei with masses between the ones corresponding to proton and iron nuclei.

Of course, there are other many other possibilities for the primary particle, apart from being an hadron. Photons and neutrinos are alternatives that need to be studied as well because of their importance from the theoretical point of view.

The case of photons is particularly interesting, specially at the highest energies. In normal conditions, a photon entering the atmosphere produces a electron-positron pair that in turn generate a electromagnetic shower. When the energy of the primary photon is very large (greater than $10^{19} \mathrm{eV}$ ), the cross section for pair production reduces progressively with the primary energy as a consequence of the so called LPM effect [15]. The LPM effect can modify significantly the typical longitudinal development of an electromagnetic shower, as discussed in detail in reference [15]: Photon showers can develop deeper in the atmosphere, and the shower to shower fluctuations are substantially larger in comparison with the no LPM case.

But the story does not end with this: a highest energy primary photon can interact with the Earth's magnetic field prior to reach the atmosphere, generating an electron-positron pair which in turn will emit secondary bremsstrahlung photons, all this giving rise to an electromagnetic shower commonly named preshower to emphasize that it develops before the particles can hit the atmosphere. When the preshower particles enter the atmosphere they evolve like normal electromagnetic 
particles. The mean energy per particle is sensibly lower than the primary energy, and therefore the LPM effect is no more significant. As a consequence these preconverted showers are less fluctuating than the unconverted ones, and so more difficult to distinguish from showers initiated by hadrons.

The accuracy of composition determinations making use of the longitudinal shower development is always limited by the fact that these determinations must be done via comparisons with simulations that depend on models still not completely established. This fact shows up in figure 2 where $X_{\max }$ estimations coming from simulations with different hadronic models are included.

\section{HADRONIC MODELS AND AIR SHOWER SIMULATIONS}

While the electromagnetic interactions (responsible for electromagnetic sub showers, ionization, Cherenkov light production, etc.), and the weak interaction (responsible for decays of unstable secondaries) are well understood, the major uncertainties in air shower simulations arise from the hadronic interaction models. With the present theoretical understanding of soft hadronic interactions, i.e. those with a small momentum transfer, one cannot calculate interaction crosssections or particle production from first principles. Therefore, hadronic interaction models are usually a mixture of fundamental theoretical ideas and empirical parameterizations tuned to describe the experimental data available. The large extrapolation needed from experimental accelerator data to the highest energy cosmic ray interactions is also a major source of uncertainty.

The relevant range of energies of the hadronic collisions that take place during shower development spreads very widely, from about $100 \mathrm{MeV}$ up to the primary energy, i.e. $\geq 10^{20} \mathrm{eV}$. In general one needs to use separate models for low $(<100 \mathrm{GeV})$ and high $(>100 \mathrm{GeV})$ energy regimes.

Predictions from existing models are not always in complete agreement, as we have shown in previous works $[9,11$, 27]. An important source of discrepancy that has been studied recently is connected with the treatment of the so called diffractive dissociation events [28]. The diffractive collisions are characterized by a low multiplicity and fast secondary particles. They play a very important role during the development of air showers, due to the fact that they provide a way of transporting substantial amounts of energy deep in the atmosphere, and turn into a critical factor that controls the global characteristics of the shower profile [11].

The results coming from different theoretical treatments of soft interactions are not always coincident; and they cannot be conclusively checked against experimental data because up to the present time these forward processes could not be measured with enough accuracy in collider experiments [27, 29].

An important example of such differences is shown in figure 3 where the fractions of diffractive events registered in a set of simulated collisions is plotted as a function of primary energy, in the case of proton primaries. The very significant difference between the QGSJET and SIBYLL cases

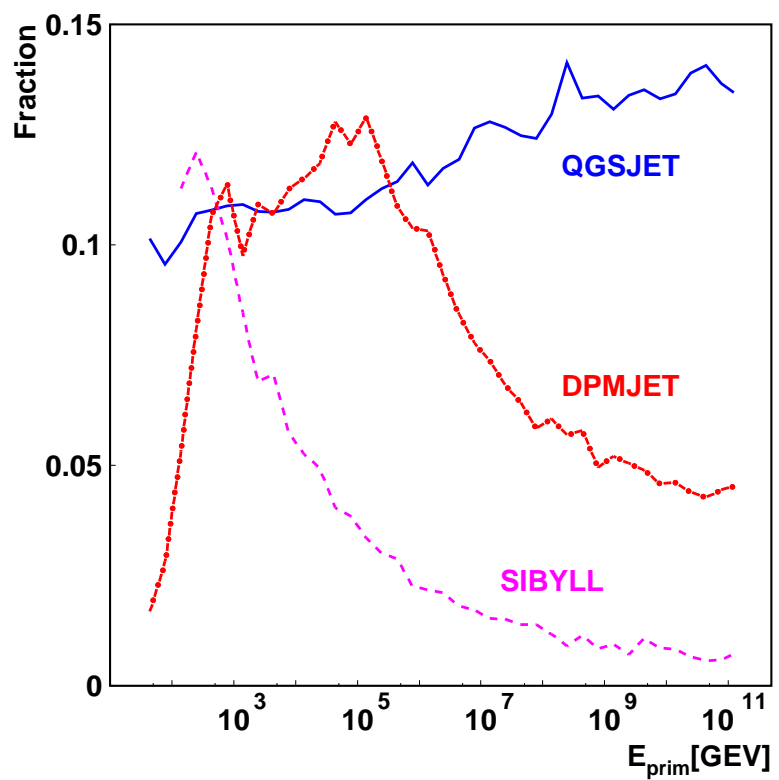

FIG. 3: Fraction of diffractive events versus primary energies for the case of proton-air collisions.

is one of the outstanding features of this plot: these results indicate that in QGSJET the ratio between the diffractive and total cross sections does not suffer substantial variations in the whole range of energies considered (from $30 \mathrm{GeV}$ to 100 $\mathrm{EeV}$ ), while the corresponding cross section ratio for SIBYLL presents a completely different behavior, decreasing as the primary energy increases. In between of these two completely different behaviors we can place the DPMJET case, characterized by a diffractive probability similar to QGSJET, for primary energies up to $10^{15} \mathrm{eV}$ approximately, and then decreasing continuously for larger primary energies.

A simple theoretical analysis, supported by the experimental evidence available, indicates that the asymptotic behavior of the QGSJET fraction of diffractive events could be mistaken [28]. A new version (QGSJETII) of this model that has been made available recently, seems not to suffer from this problem. We are currently investigating in deep the characteristics of this modified hadronic package and will publish our results elsewhere [30]

\section{FINAL REMARKS}

In this talk we tried to review a number of currently active aspects of cosmic ray physics research, both experimental and theoretical.

The Auger Observatory is, at present, the largest experimental effort ever attempted to try to obtain data accurate enough to solve the basic questions about the highest energy cosmic rays. It is expected that the data that will be taken in the following years will allow us to determine whether or not these particles hit the Earth isotropically; to obtain an accurate energy spectrum indicating clearly either that an end to 
the spectrum (the GZK cutoff) exists, or showing with enough statistics how many ultra-GZK particles are we receiving at the Earth; and, if possible, to give an idea of what those particles are.

The experimental data alone will surely not be sufficient to answer the questions we want to answer. A long and involved analysis is needed to interpret them, including unavoidable comparisons with simulated data. For this reason, computer simulations of cosmic ray interactions remain a central issue, with some problems that are still open.
[1] For a review of the early history of cosmic ray physics see, for example, M. S. Longair, High Energy Astrophysics, vol. 1, Cambridge University Press (1994).

[2] P. Auger, Rev. Mod. Phys., 11, 288 (1939).

[3] J. Linsley, Phys. Rev. Lett., 10, 146 (1963).

[4] The Pierre Auger Observatory Collaboration, Nucl. Instr. and Meth. A, 523, 50 (2004); see also www.auger.org .

[5] P. Mantsch, for the Pierre Auger Collaboration, astro$\mathrm{ph} / 0604114$ (2006).

[6] The Pierre Auger Collaboration (Presented by P. Sommers), First Estimate of the Primary Cosmic Ray Energy Spectrum above $3 \mathrm{EeV}$ from the Pierre Auger Observatory, Proc. 29th ICRC (Pune), 1, 124 (2005)

[7] The Pierre Auger Collaboration (Presented by A. LetessierSelvon), Anisotropy Studies Around the Galactic Center at EeV Energies with Auger Data, Proc. 29th ICRC (Pune), 1, 119 (2005).

[8] The Pierre Auger Collaboration (Presented by M. Risse), Upper limit on the primary photon fraction from the Pierre Auger Observatory, Proc. 29th ICRC (Pune), 1, 107 (2005).

[9] S. J. Sciutto, Proc. 27th ICRC (Hamburg), 1, 237 (2001) (Copernicus Gesellschaft, Hamburg, 2001); see also www.fisica.unlp.edu.ar/auger/aires .

[10] S. J. Sciutto, J. Knapp, D. Heck, Proc. 27th ICRC (Hamburg), 1, 526 (2001).

[11] J. Knapp, D. Heck, S. J. Sciutto, M. T. Dova, M. Risse, Astrop. Phys., 19, 77 (2003).

[12] R. Engel, T. K. Gaisser, T. Stanev, Proc. 26th ICRC (Utah), 1, 415 (1999) (AIP Conference Proceedings, New York (1999)).

[13] N. N. Kalmykov, S. S. Ostapchenko, A. I. Pavlov, Nucl. Phys. B (Proc. Suppl.), 52B, 17 (1997); D. Heck, Proc. 27th ICRC (Hamburg), 1, 433 (2001) (Copernicus Gesellschaft, Hamburg, 2001)

[14] S. Ostapchenko, Nucl. Phys. Proc. Suppl., B151, 143 (2006).

[15] A. N. Cillis, H. Fanchiotti, C. A. García Canal, S. J. Sciutto, Phys. Rev. D, 59, 113012 (1999).

[16] A. N. Cillis, Sciutto, S. J., Phys. Rev. D, 64, 013010 (2001).
[17] A. N. Cillis, S. J. Sciutto, J. Phys. G, 26, 309 (2000).

[18] N. Sakaki, et al., Proc 27th ICRC (Hamburg), 1, 329 (2001).

[19] X. Bertou, P. Billoir, O. Deligny, C. Lachaud, A. LetessierSelvon, Astropart. Phys., 17, 183 (2002).

[20] The Pierre Auger Collaboration (Presented by P. Guia), Statistical and systematic uncertainties in the event reconstruction and $S(1000)$ determination by the Pierre Auger surface detector, Proc. 29th ICRC (Pune), 1, 103 (2005).

[21] D. Barnhill et al., for The Pierre Auger Collaboration (Presented by P. Bauleo), Measurement of the Lateral Distribution Function of UHECR Air Showers with the Pierre Auger Observatory, Proc. 29th ICRC (Pune), 1, 114 (2005).

[22] The Pierre Auger Collaboration (Presented by L. Nellen), Detection of Very Inclined Showers with the Auger Observatory, Proc. 29th ICRC (Pune), 1, 115 (2005).

[23] The Pierre Auger Collaboration (Presented by J. A. Bellido), Performance of the Fluorescent Detectors of the Pierre Auger Observatory, Proc. 29th ICRC (Pune), 1, 113 (2005).

[24] The Pierre Auger Collaboration (Presented by M. A. Mostafá), The Hybrid Performance of the Pierre Auger Observatory, Proc. 29th ICRC (Pune), 1, 125 (2005).

[25] The Pierre Auger Collaboration (Presented by F. Nerling, J. Blümer, R. Engel, M. Unger), Impact of a new Cherenkov light parameterisation on the reconstruction of shower profiles from Auger hybrid data, Proc. 29th ICRC (Pune), 1, 110 (2005).

[26] R. Cester et al., for The Pierre Auger Collaboration (Presented by M. Roberts), Atmospheric aerosol monitoring at the Pierre Auger Observatory, Proc. 29th ICRC (Pune), 1, 136 (2005).

[27] S. J. Sciutto, in Proc. of the X Mexican School of Particles and Fields, AIP Conference Proceedings vol. 670, New York (2003).

[28] R. Luna, A. Zepeda, C. A. García Canal, S. J. Sciutto, Phys. Rev. D, 70, 114034 (2004).

[29] R. Engel, Nucl. Phys. B (Proc. Suppl.), 122, 40 (2003).

[30] S. J. Sciutto, work in progress. 